\title{
The role of ursodeoxycholic acid on cholestatic hepatic fibrosis in infant rats
}

\author{
NING TANG ${ }^{1}$, YAPING ZHANG ${ }^{1}$, QINGHONG LIANG ${ }^{1}$, ZEYU LIU $^{1}$ and YINGPING SHI ${ }^{2}$ \\ ${ }^{1}$ Department of Pediatrics, Third Hospital of Hebei Medical University, Shijiazhuang, Hebei 050051; \\ ${ }^{2}$ Department of Pathology, Tianjin Nankai Hospital, Tianjin 300100, P.R. China
}

Received April 24, 2017; Accepted November 8, 2017

DOI: $10.3892 / \mathrm{mmr} .2017 .8284$

\begin{abstract}
The aim of the present study was to identify the impact of ursodeoxycholic acid (UDCA) on liver function and fibrosis markers in infant rats by establishing a cholestatic-induced hepatic fibrosis model. $\alpha$-naphthylisothiocyanate (ANIT) was administrated by gavage to induce cholestatic hepatic fibrosis in infant rats. UCDA treatment was performed to assess its impact on biochemical indicators of liver function, four serum biomarkers of hepatic fibrosis, hepatic fibrosis indices in liver tissues and the pathology of liver tissues. Colorimetric assays and biochemical assays based on the initial rate method were performed to determine the levels of liver function markers in the serum, whereas the serum biomarkers of hepatic fibrosis were measured via radioimmunoassay. Sections of liver tissue were harvested and stained with hematoxylin-eosin or picric acid-Sirius red, and subjected to immunohistochemical staining to analyze liver pathology. All indicators of liver function, except for cholinesterase, were significantly higher in the ANIT model than in the control group $(\mathrm{P}<0.01) . \gamma$-glutamyl transpeptidase and total bile acids of the UDCA treatment group were significantly lower than the ANIT model $(\mathrm{P}<0.05)$; whereas no significant differences were observed in alanine aminotransferase, aspartate aminotransferase, total bilirubin, direct bilirubin and indirect bilirubin between the two groups. Serum laminin protein (LN) and type-IV collagen (cIV) in the UDCA treatment group were significantly lower than in the ANIT model $(\mathrm{P}<0.01)$; whereas no significant differences were observed in hyaluronic acid and type-III procollagen between the two groups. Liver LN and cIV in the UDCA treatment group were significantly lower than in the ANIT model $(\mathrm{P}<0.01)$. The degree of hepatic fibrosis in the UDCA treatment group was significantly lower than in the ANIT model $(\mathrm{P}<0.01)$. The results of the present study demonstrated that
\end{abstract}

Correspondence to: Dr Yaping Zhang, Department of Pediatrics, Third Hospital of Hebei Medical University, 139 Ziqiang Road, Shijiazhuang, Hebei 050051, P.R. China

E-mail: yapingzhang@aliyun.com

Key words: ursodeoxycholic acid, cholestasis, hepatic fibrosis, liver functions, hepatic fibrosis markers, infant rats
UDCA is able to reduce $\mathrm{LN}$ and cIV in serum and protect liver tissues against hepatic fibrosis.

\section{Introduction}

Cholestasis in infants is a relatively common infant disease, with an incidence of $1 / 2,500$ among newborns worldwide $(1,2)$. Delayed treatment of cholestasis may lead to accumulation of bile salts and other toxic components of bile in hepatocytes and throughout the body, resulting in cholestatic hepatic fibrosis and even liver cirrhosis; as such, it is a notable cause of infant mortality and disability (3). For a variety of reasons, including infection, poisoning, autoimmune diseases, and genetic defects, metabolic abnormalities induce hepatocyte dysfunction, or bile duct obstruction and reduced bile flow, which then induce cholestasis in infants. Early diagnosis of cholestasis, and timely and effective treatment measures are required to cease disease progression in infants $(4,5)$. Due to the nature of infant growth and development, treatment for cholestasis in infants has been difficult for pediatricians (6). Therefore, it is necessary to develop a reliable pharmacological agent with good efficacy, few side effects and that is suitable for children.

In recent years, with advances in medical technology, a number of underlying mechanisms of cholestasis have been identified $(2,6,7)$. Diagnosis and treatment of cholestasis in infants has made substantial progress; however, the overall diagnosis and treatment for the disease in China lag behind those of other countries (8). A number of previous studies have demonstrated that ursodeoxycholic acid (UDCA) is effective for the treatment of cholestasis (9-11), and it has also been used as a treatment for many types of intrahepatic cholestasis (12). UDCA has been found to possibly replace the toxic hydrophobic bile salts in enterohepatic circulation, inhibit the absorption of other bile salts in the ileum, improve the mobility of the basolateral membrane of the hepatic sinusoid and hepatic-bile capillary membrane (13), protect the membranes of hepatocytes, enhance the excretory function of hepatocytes by improving the gallbladder, and protecting hepatocytes to inhibit the pathological process of liver fibrosis and cirrhosis (14). $\alpha$-naphthylisothiocyanate (ANIT) has been extensively used for establishing liver fibrosis models. The present study established a reversible acute ANIT-induced cholestatic hepatic fibrosis model in infant rats and used UDCA to treat the disease to simulate UDCA treatment for 
cholestatic hepatic fibrosis in infants using the in vivo model. Through detecting the biochemical markers for liver function, four serum markers for hepatic fibrosis [hyaluronic acid (HA), type-III procollagen (PCIII), laminin protein (LN) and type-IV collagen (cIV)], hepatic fibrosis markers in liver disease, and pathomorphological changes in different groups of the rat model, the therapeutic effect of UDCA on cholestatic hepatic fibrosis in infant rats was evaluated, with the aim of identifying an effective treatment for early intervention of the disease in infants.

\section{Materials and methods}

Experimental animals and reagents. A total of 49 3-week old healthy Sprague Dawley (SD) rats (clean grade; weight, 50-70 g; 24 male, 25 female) were purchased from the Experimental Animal Center of Hebei Province (Hebei Medical University, Shijiazhuang, Hebei, China; certificate nos. 1307128, 1308020 and 1308039). Rats were housed in the Experimental Animal Center at the Third Hospital of Hebei Medial University (Shijiazhuang, Hebei, China) under standard conditions (temperature, $25 \pm 1^{\circ} \mathrm{C}$, humidity $\sim 70 \%, 12$-h light/dark cycle), with ad libitum access to standard diet and water. Rats were randomly divided into three groups: ANIT model group $(n=20)$, UDCA treatment group $(n=19)$ and control group $(n=10)$. ANIT was purchased from Sigma-Aldrich (Merck KGaA, Darmstadt, Germany). UDCA was purchased from Losan Pharma GmbH (Neuenburg, Germany) and prepared as a $1.6 \%$ UDCA solution using $0.9 \%$ sodium chloride. All procedures involving animals were reviewed and approved by the Institutional Animal Care and Use Committee of the Third Hospital of Hebei Medical University (certificate no. A2013-002-1).

Animal modeling. Different ANIT doses (50, 75 and $100 \mathrm{mg}$ ) and different time points were selected to build a cholestatic hepatic fibrosis model, and it was identified that $75 \mathrm{mg} / \mathrm{kg} / \mathrm{kg}$ $1 \%$ ANIT for 2 days (once daily) was best to build a good cholestatic hepatic fibrosis model. A dose of $75 \mathrm{mg} / \mathrm{kg}$ ANIT has recently been demonstrated to induce cholestatic hepatic fibrosis (15). Rats in the present study in the ANIT model group were administrated $75 \mathrm{mg} / \mathrm{kg} 1 \%$ ANIT via gavage on the fourth day of experiment; rats in the UDCA treatment group were administered $100 \mathrm{mg} / \mathrm{kg}$ UCDA solution daily from the second day of the experiment and on the fourth day of experiment administration of $75 \mathrm{mg} / \mathrm{kg} 1 \%$ ANIT was performed via gavage until sacrifice of all the rats on the sixth day of the experiment. Rats in the control group received normal diet, as above, prior to decapitation. Rats in all groups were fasted for $12 \mathrm{~h}$ prior to and following gavage, with ad libitum access to water to ensure adequate and even drug absorption. At the time of sacrifice, the weight range of rats was 50-60 g. All rats were sacrificed via decapitation following anesthesia via intraperitoneal injection of $0.1 \mathrm{ml} / 200 \mathrm{~g}$ ketamine hydrochloride/xylazine (Sigma-Aldrich; Merck KGaA).

Experimental methods. According to the results of the preliminary experiments, rats were administered the appropriate treatment following $24 \mathrm{~h}$ acclimating. Rats were sacrificed at 2 days following ANIT gavage and 4-5 ml blood was collected, frozen at $-20^{\circ} \mathrm{C}$ and used for the detection of serum biomarkers of hepatic fibrosis (HA; PCIII; LN and cIV) and liver functions [alanine aminotransferase (ALT), aspartate aminotransferase (AST), $\gamma$-glutamyl transpeptidase $(\gamma-\mathrm{GT})$, total bilirubin (TBIL), direct bilirubin (DBIL), indirect bilirubin (IBIL), cholinesterase (CHE) and total bile acids (TBA)]. Following blood collection, the peritoneal cavities of rats were opened to dissect $1.0 \times 0.8 \times 0.3 \mathrm{~cm}$ liver tissue samples from the right hepatic lobe, followed by fixing the tissues in $10 \%$ neutral formalin at temperature $25^{\circ} \mathrm{C}$ for $4-6 \mathrm{~h}$ at least, conventional dehydration, paraffin embedding and sectioning at 4-6 $\mu \mathrm{m})$.

Liver function. A total of 43 serum samples fitted in the experimental requirements from the 49 samples (6 rats failed to provide a sufficient blood sample and were excluded): 8 samples from the control group, 18 samples from the ANIT model group and 17 samples from the UCDA treatment group. Liver function indices (ALT, AST, TBIL, DBIL, IBIL, $\gamma$-GT, CHE, and TBA) were measured using assay reagents (Aspartate Aminotransferase kit no. 12-0763, Alanine Aminotransferase kit no. 12-0739, Total Bilirubin kit no. 12063017, Direct Bilirubin kit no. 12061821, $\gamma$-glutamyl transpeptidase kit no. 12-0777, Cholinesterase kit no. 12-0635 and Total Bile Acids kit no. 12-0652) purchased from Johnson \& Johnson Medical (Shanghai), Ltd. (Shanghai, China). IBIL was calculated as TBIL-DBIL. An Olympus AU5400 automatic biochemical analyzer (Olympus Corporation, Tokyo, Japan) was used for detection of function indices. Rat blood samples $(2-3 \mathrm{ml})$ were placed in pro-coagulation tubes, which were then centrifuged at $1,006 \mathrm{x} \mathrm{g}$ at $4^{\circ} \mathrm{C}$ for $3 \mathrm{~min}$ to isolate serum for ALT, AST, $\gamma$-GT, CHE, and TBA biochemical assays based on the velocity method, and TBIL, DBIL, and IBIL for biochemical assays based on colorimetry $(8,16)$. Some rats failed to provide a sufficient blood sample, so the case for liver function was less.

Serum biomarkers of hepatic fibrosis. Chose 43 serum samples fitted in the experimental requirements from the 49 samples, 8 samples from the control group, 18 samples from the ANIT model group and 17 samples from the UCDA treatment group. The serum biomarkers (HA, PCIII, LN, and cIV) of hepatic fibrosis were detected via radioimmunoassay (RIA). Rat blood samples (2-3 ml) were placed in pro-coagulation tubes, and centrifuged at $1,006 \mathrm{x} \mathrm{g}$ at $4^{\circ} \mathrm{C}$ for $3 \mathrm{~min}$ to isolate serum. To detect the serum biomarkers of hepatic fibrosis, radioimmunoassay kits (Hyaluronic acid kit, 012-1186; Type-III procollagen kit, 012-1335; Laminin protein kit, 012-1219; and Type-IV collagen kit, 12-1452; Shanghai Haiyan Biotechnology Institute, Shanghai, China) and an XH-6020 $\gamma$-RIA counter (Xi'an Nuclear Instrument Factory, Xi'an, China) were used according to the manufacturers' protocols $(8,16)$.

Hematoxylin and eosin $(H \& E)$ staining. Liver tissues isolated from rats were fixed in $10 \%$ neutral formalin (Zhongshan Beijing Biotechnology Co., Ltd., Beijing, China) for at least 4-6 h at $25^{\circ} \mathrm{C}$ and transferred to $70 \%$ ethanol. Individual hepatic tissues biopsy material was placed in processing cassettes, dehydrated through a serial alcohol gradient, and embedded in wax blocks. Sections were cut at 4-6 $\mu \mathrm{m}$ and dewaxed in xylene, rehydrated through decreasing concentrations of 
ethanol, and washed in PBS. The sections were then stained with hematoxylin for 4-5 min and with eosin for 1-2 min at $25^{\circ} \mathrm{C}$ (Zhongshan Beijing Biotechnology Co., Ltd.). Stained liver sections were observed and photographed under light microscopy (magnification, x200; BX53-p; Olympus Corporation) (16).

Picric acid-sirius red staining. Liver tissues isolated from rats were fixed in $10 \%$ neutral formalin (Zhongshan Beijing Biotechnology Co., Ltd., Beijing, China) for at least 4-6 h at $25^{\circ} \mathrm{C}$ and transferred to $70 \%$ ethanol. Individual hepatic tissues biopsy material was placed in processing cassettes, dehydrated through a serial alcohol gradient, and embedded in wax blocks. Sections were cut at 4-6 $\mu \mathrm{m}$ and dewaxed in xylene, rehydrated through decreasing concentrations of ethanol, and washed in PBS. The sections, mounted on glass slides, were stained with picric acid-Sirius red $(0.1 \%$ Sirius red in saturated aqueous picric acid; Zhongshan Beijing Biotechnology Co., Ltd.) for $5 \mathrm{~min}$ at $25^{\circ} \mathrm{C}$ to detect hepatic fibrosis. Stained sections were examined with polarizing microscopy at magnifications of x4, x10, x100 and x400(16).

Immunohistochemical staining. A total of 46 serum samples were selected from the 49 samples, 10 sample from the control group, 13 samples from the ANIT group and 13 samples from the UCDA group, respectively. For the immunohistochemical staining of cIV and LN, primary antibodies were purchased from Wuhan Boster Biological Technology, Ltd. (Wuhan, China; Laminin A03522 and cIV BA3585-2), and the Goat Anti-Rabbit secondary antibody was purchased from OriGene Technologies, Inc. (Beijing, China). Dilution of all antibodies in immunohistochemical staining was 1:150. PBS was used to replace primary antibodies as a negative control. Following incubation with goat serum for $10 \mathrm{~min}$ at room temperature, samples were incubated with primary antibodies (rabbit-anti-rat) for $30 \mathrm{~min}$ at $37^{\circ} \mathrm{C}$, washed with PBS 3 times for $3 \mathrm{~min}$ each wash and then incubated with the secondary antibody at $37^{\circ} \mathrm{C}$ for $20 \mathrm{~min}$. The samples were washed with PBS 3 times for 3 min each wash and stained with DAB for $5 \mathrm{~min}$ at room temperature. The slides were washed, dehydrated as above, and observed using an Olympus BX51 optical microscope (Olympus Corporation). Positive expression of cIV and LN presented as light yellow or brown granules or linear deposition was localized in the cytoplasm, membrane or interstitial regions. Images were acquired of the tissue sections from 5 randomly selected visual fields, using magnification, $\mathrm{x} 10$ for cIV staining and magnification, x20 for LN staining. Image Pro-Plus 6.0 software (Media Cybernetics, Inc., Rockville, MD, USA) was used to quantitatively analyze the integrated optical density in each of the 5 randomly selected visual fields per tissue section. The following formula was used to obtain the integrated optical density (IOD) of the positively-stained region following the removal of the background value: IOD of the positively stained region/area of positively stained region-IOD of the negatively stained region/area of negatively stained region. The mean of the results of each tissue section from the 5 randomly selected visual fields was then calculated.

Pathological grading of hepatic fibrosis. A total of 47 serum samples were selected from the 49 samples, 8 samples from the control group, 20 samples from the ANIT model group and 19 samples from the UCDA treatment group. Conventional H\&E-stained sections (as above) from different samples were used to observe liver cell degeneration, the degree of collagen fiber proliferation and differences in morphology. Pathological grading of hepatic fibrosis was performed in accordance with Knodell scores (17), staging criteria, and quantitative and semi-quantitative scoring methods as follows: 0 points, normal hepatocyte morphology, no lesion, and no marked collagen fiber proliferation in hepatic lobules; 1 point, $<25 \%$ degeneration of total hepatocytes, presence of collagen fiber proliferation, small amount of fiber extension in the central vein and portal area, and tendency for fiber extension towards hepatic lobules, but no septa formation; 2 points, $25-50 \%$ degeneration of total hepatocytes, notable collagen fiber formation, thickened connective tissues in the central vein and portal area and fiber extension towards peripheral regions, formation of incomplete septa, but substantially retained lobular structure; 3 points, $50-75 \%$ degeneration of total hepatocytes, large amount of collagen fiber proliferation, some complete septa or incomplete and thickened septa to form pseudolobuli; 4 points, $>75 \%$ degeneration of total hepatocytes, complete and thick septa and a large amount of pseudolobuli (16).

Statistical analysis. SPSS 21.0 software (IBM Corp., Armonk, NY, USA) was used for statistical analysis in the present study. Data are presented as the mean \pm standard deviation. Normally distributed data with homogeneity of variance were compared using one-way-analysis of variance followed by Dunnett post hoc test for comparisons with multiple groups. Non-normally distributed date or data with heterogeneity of variance were analyzed using Mann-Whitney U test. Analysis of pathological grading indices was performed using Spearman's correlation coefficient. $\mathrm{P}<0.05$ was considered to indicate a statistically significant difference.

\section{Results}

Impact of UDCA on the liver function of infant rats with cholestatic liver fibrosis. As demonstrated in Table I, all liver function indices except for CHE were significantly increased in the ANIT model group compared with the control group $(\mathrm{P}<0.01)$, which indicated that the cholestatic liver fibrosis model was successfully established in the infant rats. In the UDCA treatment group, $\gamma$-GT and TBA were significantly lower than in the ANIT model group $(\mathrm{P}<0.05)$, whereas no significant differences were observed in ALT, AST, TBIL, DBIL, IBIL and CHE between the UDCA treatment and ANIT model groups.

Impact of UDCA on serum biomarkers of hepatic fibrosis in rats with cholestatic hepatic fibrosis. As demonstrated in Table II, serum LN and cIV were significantly higher in the ANIT model group than in the control group $(\mathrm{P}<0.05)$; whereas no significant differences were observed in serum HA and PCIII. Serum LN and cIV were significantly lower in the UDCA treatment group than in the ANIT model group $(\mathrm{P}<0.01)$; whereas no significant differences in serum HA and PCIII were observed. 
Table I. Effects of UDCA on liver function in infant cholestatic rats.

\begin{tabular}{lccc}
\hline Parameter & Control $(\mathrm{n}=8)$ & ANIT model $(\mathrm{n}=18)$ & UDCA treatment $(\mathrm{n}=17)$ \\
\hline ALT $(\mathrm{U} / \mathrm{l})$ & $47.1 \pm 15.8$ & $782.6 \pm 251.2^{\mathrm{a}}$ & $618.1 \pm 195.7$ \\
AST $(\mathrm{U} / \mathrm{l})$ & $386.4 \pm 138.8$ & $2265 \pm 524.8^{\mathrm{a}}$ & $1990.7 \pm 850.6$ \\
TBIL $(\mu \mathrm{mol} / \mathrm{l})$ & $3.0 \pm 0.9$ & $142.3 \pm 25.3^{\mathrm{a}}$ & $138.9 \pm 14.4$ \\
DBIL $(\mu \mathrm{mol} / \mathrm{l})$ & $2.5 \pm 1.1$ & $129.1 \pm 23.0^{\mathrm{a}}$ & $127.1 \pm 11.1$ \\
IBIL $(\mu \mathrm{mol} / \mathrm{l})$ & $0.5 \pm 0.3$ & $12.8 \pm 5.3^{\mathrm{a}}$ & $11.9 \pm 4.7$ \\
$\gamma-\mathrm{GT}(\mathrm{U} / \mathrm{l})$ & $0.4 \pm 3.4$ & $15.7 \pm 6.1^{\mathrm{a}}$ & $11.1 \pm 3.3^{\mathrm{b}}$ \\
CHE $(\mathrm{kU} / \mathrm{l})$ & $0.26 \pm 0.04$ & $0.25 \pm 0.05$ & $0.25 \pm 0.04$ \\
TBA $(\mu \mathrm{mol} / \mathrm{l})$ & $194.3 \pm 130.9$ & $657.5 \pm 95.1^{\mathrm{a}}$ & $608.2 \pm 63.0^{\mathrm{b}}$ \\
\hline
\end{tabular}

Data are presented as the mean \pm standard deviation. ${ }^{a} \mathrm{P}<0.01$ vs. control; ${ }^{\mathrm{b}} \mathrm{P}<0.05$ vs. ANIT model. UDCA, ursodeoxycholic acid; ANIT, $\alpha$-naphthylisothiocyanate; ALT, alanine aminotransferase; AST, aspartate aminotransferase; TBIL, total bilirubin; DBIL, direct bilirubin; IBIL, indirect bilirubin; $\gamma$-GT, $\gamma$-glutamyl transpeptidase; CHE, cholinesterase; TBA, total bile acids.

Table II. Effects of UDCA on potential biomarkers of liver fibrosis in infant cholestatic rats.

\begin{tabular}{lccccc}
\hline Group & Rats $(\mathrm{n})$ & HA $(\mathrm{ng} / \mathrm{ml})$ & PCIII $(\mathrm{ng} / \mathrm{ml})$ & $\mathrm{LN}(\mathrm{ng} / \mathrm{ml})$ & $\mathrm{cIV}(\mathrm{ng} / \mathrm{ml})$ \\
\hline Control & 8 & $1489.8 \pm 215.3$ & $40.4 \pm 1.6$ & $39.7 \pm 5.4$ & $24.9 \pm 4.1$ \\
ANIT model & 18 & $1772.7 \pm 316.9$ & $41.2 \pm 11.0$ & $48.5 \pm 8.8^{\mathrm{a}}$ & $30.8 \pm 7.4^{\mathrm{a}}$ \\
UDCA treatment & 17 & $1608.5 \pm 409.9$ & $41.6 \pm 4.6$ & $41.7 \pm 5.6^{\mathrm{b}}$ & $24.0 \pm 7.9^{\mathrm{b}}$ \\
\hline
\end{tabular}

Data are presented as the mean \pm standard deviation. ${ }^{\mathrm{a}}<0.05$ vs. control; ${ }^{\mathrm{b}} \mathrm{P}<0.01$ vs. ANIT model. UDCA, ursodeoxycholic acid; ANIT, $\alpha$-naphthylisothiocyanate; HA, hyaluronic acid; PCIII, type-III procollagen; LN, laminin protein; cIV, type-IV collagen.

Impact of UDCA on $L N$ and cIV expression in the liver tissues of the infant rats with cholestatic hepatic fibrosis. As demonstrated in Table III, the LN and cIV expression in the liver tissues of the ANIT model group were significantly higher than in the control group $(\mathrm{P}<0.01)$; whereas the LN and cIV expression in the liver tissues of the UDCA treatment group were significantly lower than in the ANIT model groups $(\mathrm{P}<0.01)$.

Impact of UDCA on the pathological grading of cholestatic hepatic fibrosis in infant rats. Table IV demonstrated that the degree of hepatic fibrosis in the liver tissues of the ANIT model group was significantly higher than in the control group $(\mathrm{P}<0.01)$, which suggested that the cholestatic hepatic fibrosis model was successfully established. The degree of hepatic fibrosis in the liver tissues of the UDCA treatment group was significantly lower than in the ANIT model group $(\mathrm{P}<0.01)$.

$H \& E$ and picric acid-Sirius red staining. H\&E and picric acid-Sirius red staining are presented in Figs. 1 and 2, respectively. In the control group, $H \& E$ staining showed a complete and clear hepatic lobule, with hepatocytes arranged neatly, which extended to peripheral regions. No abnormal proliferation of connective tissue was found in the central vein and portal area (Fig. 1A). With picric acid-Sirius red staining, tissues in the control group exhibited almost no fiber staining (Fig. 2A).
H\&E staining in the ANIT model group (Fig. 1B) indicated that the normal hepatic lobular structure was destroyed; $25-75 \%$ of liver tissues exhibited fatty degeneration, necrosis, and ballooning degeneration, with visible cholestasis in hepatocytes and small bile ducts. Notable proliferation of fibrous tissues was detected in the necrotic portal area and lobules that were accompanied by a number of neutrophil and eosinophil infiltrations. The proliferated fibrous tissues divided the hepatic lobules into pseudolobuli. With picric acid-Sirius red staining (Fig. 2B), hepatic lobules in the ANIT model group were demonstrated to have a markedly larger population of collagen fibers than the control group. Thick yellow and red fibers were distributed around or star-shaped around the central vein, distributed around the clearance of hepatic sinus and portal area, or stretched into the lobule to formed interlobular separations. A few slender green fibers were mainly distributed in the portal area, with small blood vessels and the central vein also demonstrating discontinuous distribution around the clearance of the hepatic sinus.

Compared with the ANIT model group UDCA treatment group with H\&E stained had a lower degree of hepatic fibrosis (Fig. 1C), with no significant formation of pseudolobuli and with a small amount of neutrophil and eosinophil infiltrations. Scattered necrotic debris was visible. With Sirius red-saturated picric acid stained, too, there were only few thick yellow and red fibers found in UDCA group, and the distributed of fibers was not so clear (Fig. 2C) compared with the ANIT model group. 
Table III. Effects of UDCA on immunohistochemistry of liver tissue in neonatal cholestatic rats.

\begin{tabular}{lccc}
\hline Group & Rats (n) & LN (IOD) & cIV (IOD) \\
\hline Control & 10 & $0.041 \pm 0.013$ & $0.017 \pm 0.003$ \\
ANIT model & 13 & $0.058 \pm 0.008^{\mathrm{a}}$ & $0.022 \pm 0.003^{\mathrm{a}}$ \\
UDCA treatment & 13 & $0.038 \pm 0.008^{\mathrm{b}}$ & $0.018 \pm 0.003^{\mathrm{b}}$ \\
\hline
\end{tabular}

Data are presented as the mean \pm standard deviation. ${ }^{\mathrm{a}} \mathrm{P}<0.01$ vs. control ${ }^{\mathrm{b}} \mathrm{P}<0.01$ vs. ANIT model. UDCA, ursodeoxycholic acid; IOD, integrated optical density; ANIT, $\alpha$-naphthylisothiocyanate; LN, laminin protein; cIV, type-IV collagen.

Table IV. Effects of UDCA on pathological grading of liver fibrosis in infant cholestatic rats.

\begin{tabular}{lcc}
\hline Group & Rats $(\mathrm{n})$ & Pathological score \\
\hline Control & 8 & $0.250 \pm 0.463$ \\
ANIT model & 20 & $2.100 \pm 0.641^{\mathrm{a}}$ \\
UDCA treatment & 19 & $1.000 \pm 0.577^{\mathrm{b}}$
\end{tabular}

Data are presented as the mean \pm standard deviation. ${ }^{a} \mathrm{P}<0.01$ vs. control; ${ }^{\text {b }} \mathrm{P}<0.01$ vs. ANIT model. UDCA, ursodeoxycholic acid; ANIT, $\alpha$-naphthylisothiocyanate.

Immunohistochemical staining. Immunohistochemical staining is presented in Fig. 3. A small amount of cIV and LN deposition was observed in the sinusoidal and vessel walls of liver tissues in the blank control (Fig. 3A and D), which indicated a continuous linear positive reaction. In the ANIT model and UDCA treatment groups, the brown granules of LN staining (Fig. 3E and F) were thicker and darker than the cIV staining (Fig. 3B and C). The regions of brown granule deposition were most marked in the sinusoid and portal areas, and in area of necrotic hepatocytes, showing visible necrotic cell debris. Positive cIV and LN staining was stronger in the ANIT model group (Fig. 3B and E) than in the other groups, exhibiting as coarse and linear positively stained fibers to form a fibrous septa extending into the hepatic lobules to separate the hepatic parenchyma. In the necrotic area, the positively-stained region was interwoven into a network with varying amounts of immersed inflammatory cells. Regardless of cIV and LN staining, the brown granule deposition in the positively stained region of the UDCA treatment group was markedly lower than in the ANIT model group. The portal area and hepatic parenchyma as well as the necrotic region with mild debris indicated weak and irregular thickness of linear positively staining interwoven into a network. Each type of collagen staining was not uniform and no obvious difference between the groups was observed.

\section{Discussion}

ANIT is a hepatic toxic substance that induces acute cholesteric liver injury (18). It is able to induce epithelial cell swelling and necrosis in the bile duct, which results in bile canaliculus hyperplasia and interlobular inflammatory reactions surrounding the bile ducts, which further induces cholestasis, damage to hepatic parenchyma cells and incidence of haptic fibrosis (19). ANIT-induced reversible acute intrahepatic cholestasis has previously been demonstrated as a successful animal model of intrahepatic cholestasis $(20,21)$. Results of the present study demonstrated that following $48 \mathrm{~h}$ of ANIT administration in infant SD rats, serum activities of ALT, AST, TBIL, DBIL, IBIL, and $\gamma$-GT, and TBA were significantly raised compared with the control group. In addition, LN and cIV in the serum and hepatic tissue were significantly higher in the ANIT model group than that in the control group. The structure of normal liver tissue, predominantly the portal area of the infant rats was destroyed or disappeared in the ANIT model group, suggesting that the modeling of cholestatic hepatic fibrosis was successful.

The present results also demonstrated that, following UDCA treatment, serum $\gamma$-GT and TBA of the infant SD rats were significantly reduced, compared with the ANIT model group. Serum $\gamma$-GT, which is predominantly produced by the mitochondria in hepatocytes, localizes in cytoplasm and epithelium of intrahepatic bile duct, and is excreted through bile duct into the duodenum. When extrahepatic or intrahepatic obstruction and cholestasis occur, excretion of $\gamma$-GT is blocked, and concentrations of serum $\gamma$-GT increase $(22,23)$. TBA is the part of bile acid spilled into the bloodstream and taken up by hepatocytes through intestinal reabsorption. When cholestasis or liver function is impaired, bile discharge is blocked and in the presence of secretion and/or ingestion blockage(s), TBA is also increased (24). It has previously been demonstrated that serum TBA is specific, sensitive, and stable enough to reflect liver function changes (25). A previous study by the present authors reported that serum $\gamma$-GT and TBA of infants with cholestasis were significantly higher than in healthy infants (8). Previous studies have indicated that UDCA exhibited good clinical therapeutic effects against cholestatic hepatitis, liver cirrhosis, and infant cholestatic liver diseases. For example, it has been demonstrated that UDCA significantly reduced TBA (26) and lowered hepatotoxicity of bile acids (27). However, only preliminary treatment efficacy has been reported in clinical practice $(28,29)$. Fang et al (9) recently studied the liver function indices of infants with cholestatic liver diseases that were treated by UDCA, and they demonstrated that TBIL, DBIL, ALT, $\gamma$-GT and TBA were significantly reduced when compared with pretreatment levels. In the present study, AST level were higher than that in previous studies $(9,30)$. A possible reason for this is that the AST level in erythrocytes is 30 times higher than that in the serum, whereas the ALT level in erythrocytes is only 7 times higher than that in the serum. It is possible that hemolysis may have led to high AST levels when rats were decapitated to harvest blood; which is a limitation of the present study.

Hepatic fibrosis is a response reaction to excessive collagen and proteoglycan deposition in the liver, and liver injury caused by other extracellular matrix macromolecules (31). LN belongs to a structural glycoprotein that was positively correlated with the degree of activity of hepatic fibrosis and portal vein pressure. It has high sensitivity in the diagnosis of hepatic fibrosis (32). Significant deposition of LN in hepatic sinusoids 

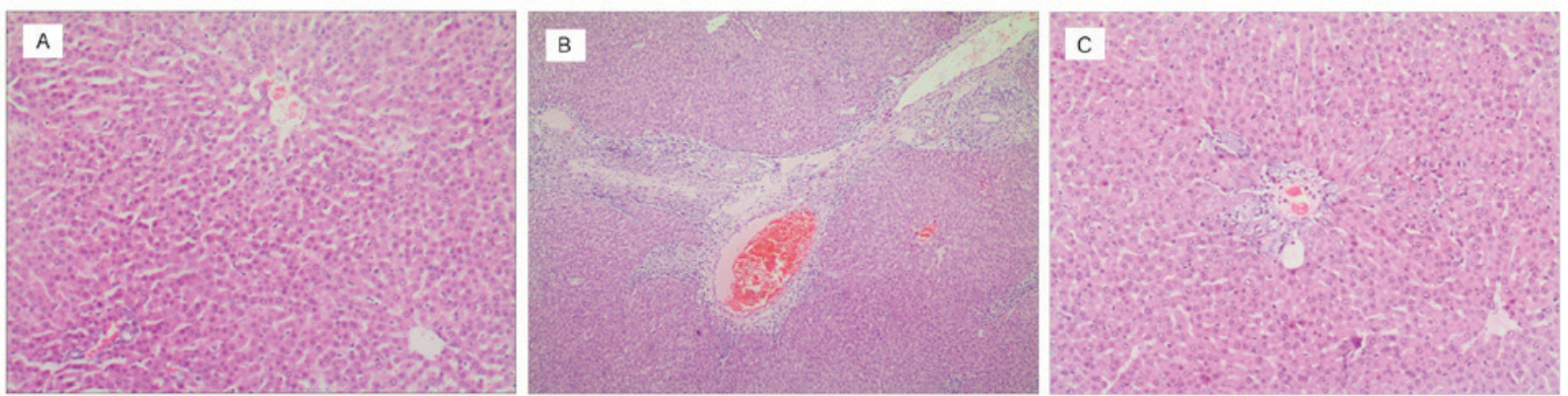

Figure 1. H\&E staining of hepatic tissues. (A) Hepatic lobules in the control group stained with H\&E. Hepatic lobules were holonomic and clear, and hepatic cells were arranged in funicular form. Almost no proliferation of fiber tissue was observed. Magnification of original image, x100. (B) Liver of ANIT model group stained with H\&E. Fatty degeneration, necrosis and ballooning degeneration was observed in $25-75 \%$ of liver tissues, fibrosis was notable present and fibrous septum divided necrosis hepatic lobules into pseudolobules. Magnification of original image, $x 40$. (C) Liver of ursodeoxycholic acid treatment group stained with H\&E. The majority of rats exhibited a lower degree of hepatic fibrosis compared with the ANIT model group, with no marked formation of pseudolobuli and with a small amount of neutrophil and eosinophil infiltrations. Scattered necrotic debris was visible. Magnification of original image, $x 100$. H\&E, hematoxylin-eosin; ANIT, $\alpha$-naphthylisothiocyanate.
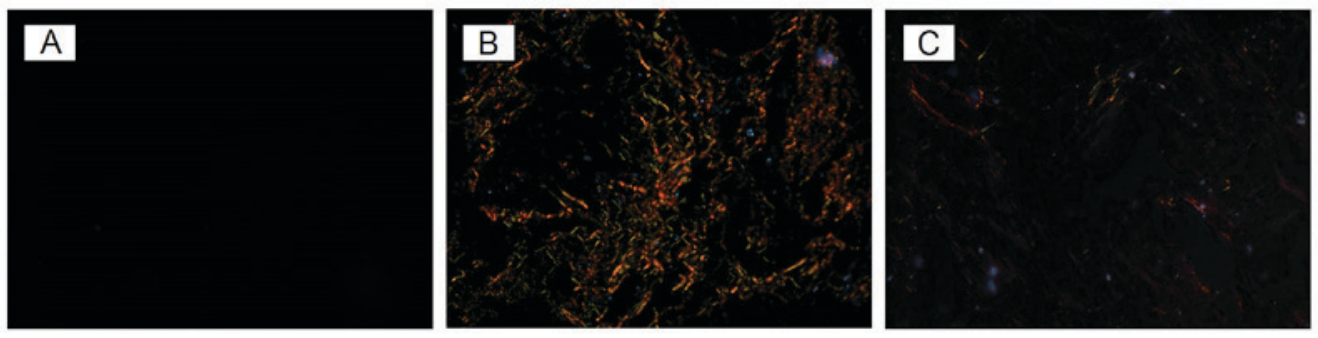

Figure 2. Sirius red-saturated picric acid staining of hepatic tissues. (A) Hepatic lobules in the control group stained with Sirius red-saturated picric acid Almost no staining was observed. Magnification of original image, x400. (B) Liver of $\alpha$-naphthylisothiocyanate model group stained with Sirius red-saturated picric acid. Thick yellow and red fibers were distributed around or star shaped around the central vein, and distributed around the clearance of hepatic sinus and portal area, with some stretched into the lobule and forming interlobular separations. A few slender green fibers were observed, predominantly distributed in portal area, small blood vessels and central vein, and also demonstrated discontinuous distribution around the clearance of the hepatic sinus. Magnification of original image, $\mathrm{x}$ 400. (C) Liver of ursodeoxycholic acid treatment group stained with Sirius red-saturated picric acid. A very small number of thick yellow and red fibers were observed, and the distribution of fibers was not well-defined. Magnification of original image, $\mathrm{x} 400$.
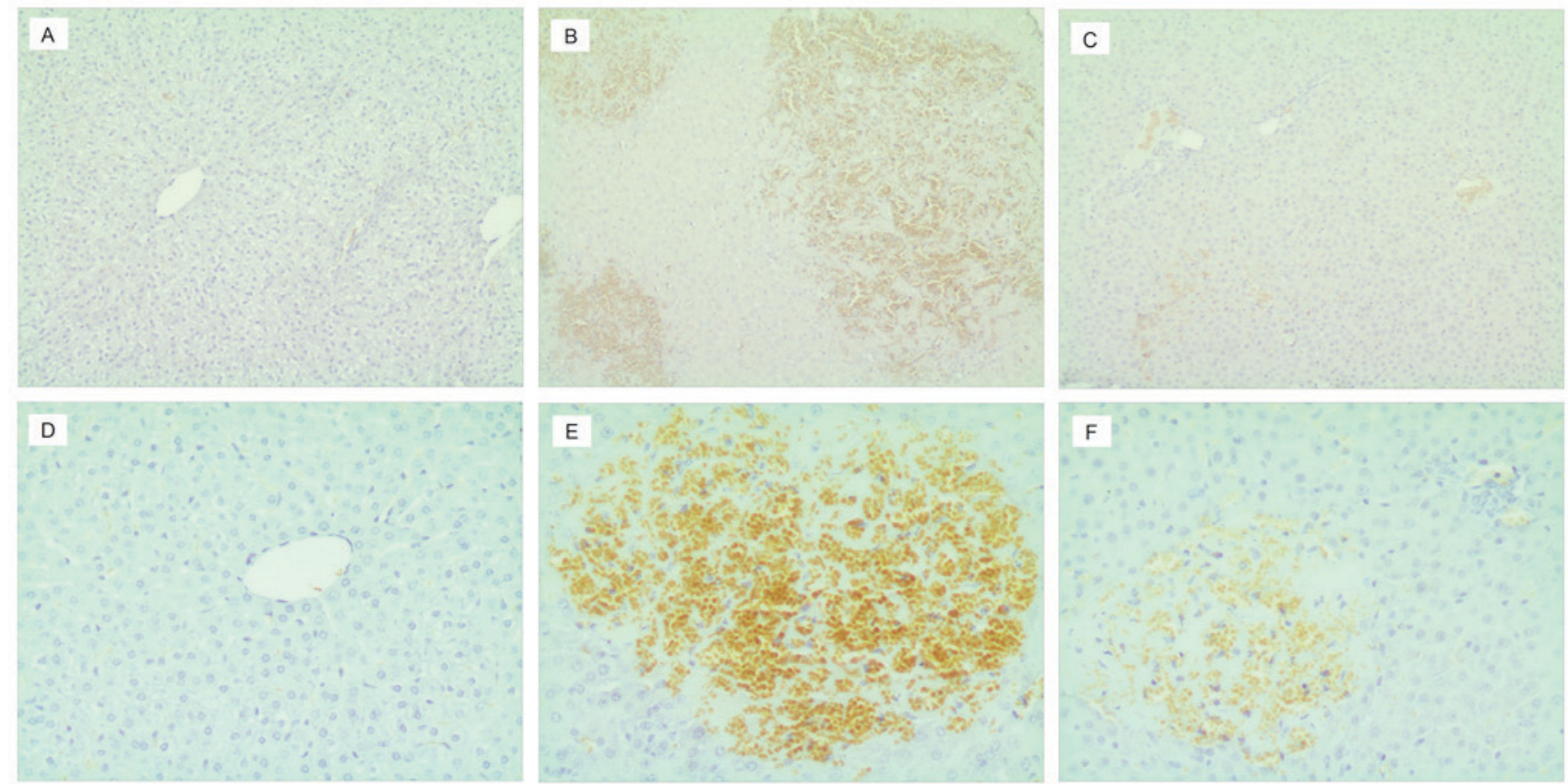

Figure 3. Immunohistochemical staining of hepatic tissues (magnification, $\mathrm{x} 400$ ). (A) cIV of hepatic tissues in the control group with immunohistochemical staining. (B) cIV of hepatic tissues in the ANIT model group with immunohistochemical staining. (C) cIV of hepatic tissues in the UDCA treatment group with immunohistochemical staining. (D) LN of hepatic tissues in the control group with immunohistochemical staining. (E) LN of hepatic tissues in the ANIT model group with immunohistochemical staining. (F) LN of hepatic tissues in the UDCA treatment group with immunohistochemical staining. cIV, type-IV collagen; ANIT, $\alpha$-naphthylisothiocyanate; UDCA, ursodeoxycholic acid; LN, laminin protein. 
and its excretion into the bloodstream typically occurs in acute hepatic fibrosis, resulting in elevated serum LN levels. cIV, as the primary component constituting the basement membrane, is a marker for early hepatic fibrosis and is considered as an indicator for collagen production rather than collagen degradation (33). cIV and LN are positively correlated with the degree of hepatic fibrosis (9). HA is typically ingested and decomposed by sinusoidal endothelial cells. When liver tissue is damaged, HA synthesized by hepatic stellate cells is increased, and its degradation in sinusoidal endothelial cells is decreased, resulting in elevated serum HA (34). HA is able to accurately and sensitively indicate hepatocyte damages and the quantity of intrahepatic fiber production (35-37). PCIII is a precursor of type III collagen that is not only valuable in the early diagnosis of hepatic fibrosis, but is also beneficial for predicting the prognosis of chronic liver disease. The serum PCIII quantity is consistent with the degree and activity of hepatic fibrosis (38). In the present study, LN and cIV expressions in the serum and liver tissues of the infant rats with cholestatic hepatic fibrosis following UDCA treatment were significantly reduced, and the UDCA treated rats exhibited marked improvements in their fibrotic liver tissues. No significant differences in serum HA and PCIII were observed between the UDCA treatment group and the ANIT model group, confirming that UDCA is able to reduce $\mathrm{LN}$ and $\mathrm{cIV}$, thereby playing a role in anti-hepatic fibrosis.

Recently, the molecular mechanisms underlying the effects of UDCA in cholestatic hepatic fibrosis have been the focus of an increasing number of studies $(39,40)$. Previous studies have typically focused on adult cholestasis; however, in the present study, rats with cholestatic liver fibrosis were chosen as the research object, through analyzing liver function, four serum biomarkers of hepatic fibrosis, liver fibrosis index and liver tissue pathological changes, to explore the therapeutic effect of UDCA on cholestatic liver fibrosis in rats. The aim was to identify an effective and early intervention method for the treatment of cholestatic liver fibrosis in infants. Limitations of the present study include the short duration of modeling and treatment, and rats were administrated with UDCA prior to ANIT modeling to achieve more observable effects of UDCA; therefore, results cannot completely reflect the anti-hepatic fibrosis effect of UDCA. It is hoped in a future study to clarify the therapeutic effect of UDCA on children with cholestatic liver disease, by improving the modeling method, the experimental design and increasing the animal sample size. Although some experimental results still require validation, the present study suggested that UDCA was able to improve liver function through the reduction of serum $\gamma$-GT and TBA, and improved hepatic fibrosis through the reduction of $\mathrm{LN}$ and cIV expression to prevent liver cirrhosis.

Future studies should be performed to investigate the role of UDCA in HSCs in children with cholestatic liver fibrosis, in order to elucidate the mechanism of UDCA in resisting hepatic fibrosis.

\section{References}

1. Fischler B and Lamireau T: Cholestasis in the newborn and infant. Clin Res Hepatol Gastroenterol 38: 263-267, 2014.

2. Catzola A and Vajro P: Management options for cholestatic liver disease in children. Expert Rev Gastroenterol Hepatol 11: 1019-1030, 2017.
3. Hernandez-Gea V and Friedman SL: Pathogenesis of liver fibrosis. Annu Rev Pathol 6: 425-456, 2011.

4. Lindor KD, Gershwin ME, Poupon R, Kaplan M, Bergasa NV and Heathcote EJ; American Association for Study of Liver Diseases: Primary biliary cirrhosis. Hepatology 50: 291-308, 2009.

5. European Association for the Study of the Liver: EASL clinical practice guidelines: Management of cholestatic liver diseases. J Hepatol 51: 237-267, 2009.

6. Orso G, Mandato C, Veropalumbo C, Cecchi N, Garzi A and Vajro P: Pediatric parenteral nutrition-associated liver disease and cholestasis: Novel advances in pathomechanisms-based prevention and treatment. Dig Liver Dis 48: 215-222, 2016.

7. Qiu YL, Gong JY, Feng JY, Wang RX, Han J, Liu T, Lu Y, Li LT, Zhang MH, Sheps JA, et al: Defects in myosin VB are associated with a spectrum of previously undiagnosed low $\gamma$-glutamyltransferase cholestasis. Hepatology 65: 1655-1669, 2017.

8. Tang N, Zhang Y, Liu Z, Fu T, Liang Q and Ai X: Correlation analysis between four serum biomarkers of liver fibrosis and liver function in infants with cholestasis. Biomed Rep 5: 107-112, 2016.

9. Fang YQ, Lv DX, Jia W, Li J, Deng YQ, Wang Y, Yu M and Wang GQ: Case-control study on prednisolone combined with ursodeoxycholic acid and azathioprine in pure primary biliary cirrhosis with high levels of immunoglobulin $G$ and transaminases: Efficacy and safety analysis. Medicine (Baltimore) 93: e104, 2014.

10. Rudic JS, Poropat G, Krstic MN, Bjelakovic G and Gluud C: Ursodeoxycholic acid for primary biliary cirrhosis. Cochrane Database Syst Rev 12: CD000551, 2012

11. Simić D, Milojević I, Bogićević D, Milenović M, Radlović V, Drasković B, Benka AU, Sindjić S and Maksimović R: Preventive effect of ursodeoxycholic acid on parenteral nutrition-associated liver disease in infants. Srp Arh Celok Lek 142: 184-188, 2014.

12. Hatano R, Kawaguchi K, Togashi F, Sugata M, Masuda S and Asano S: Ursodeoxycholic acid ameliorates intrahepatic cholestasis independent of biliary bicarbonate secretion in Vil2kd/kd mice. Biol Pharm Bull 40: 34-42, 2017.

13. Zhou WC, Zhang QB and Qiao L: Pathogenesis of liver cirrhosis. World J Gastroenterol 20: 7312-7324, 2014.

14. Mizuochi T, Kimura A, Suzuki M, Ueki I, Takei H, Nittono H, Kakiuchi T, Shigeta T, Sakamoto S, Fukuda A, et al: Successful heterozygous living donor liver transplantation for an oxysterol $7 \alpha$-hydroxylase deficiency in a Japanese patient. Liver Transpl 17: 1059-1065, 2011.

15. Wang $\mathrm{L}, \mathrm{Wu} \mathrm{G}, \mathrm{Wu} \mathrm{F}$, Jiang $\mathrm{N}$ and Lin $\mathrm{Y}$ : Geniposide attenuates ANIT-induced cholestasis through regulation of transporters and enzymes involved in bile acids homeostasis in rats. J Ethnopharmacol 20: 178-185, 2017.

16. Tang N, Zhang Y, Liu Z, Ai X and Liang Q: Correlation of four potential biomarkers of liver fibrosis with liver function and grade of hepatic fibrosis in a neonatal cholestatic rat model. Mol Med Rep 16: 415-421, 2017.

17. Knodell RG, Ishak KG, Black WC, Chen TS, Craig R, Kaplowitz N, Kiernan TW and Wollman J: Formulation and application of a numerical scoring system for assessing histological activity in asymptomatic chronic active hepatitis. Hepatology 1: 431-435, 1981.

18. Wang T, Zhou ZX, Sun LX, Li X, Xu ZM, Chen M, Zhao GL, Jiang ZZ and Zhang LY: Resveratrol effectively attenuates $\alpha$-na phthyl-isothiocyanate-induced acute cholestasis and liver injury through choleretic and anti-inflammatory mechanisms. Acta Pharmacol Sin 35: 1527-1536, 2014.

19. Connolly AK, Price SC, Connelly JC and Hinton RH: Early changes in bile duct lining cells and hepatocytes in rats treated with alpha-naphthylisothiocyanate. Toxicol Appl Pharmacol 93: 208-219, 1988.

20. Kossor DC, Meunier PC, Handler JA, Sozio RS and Goldstein RS: Temporal relationship of changes in hepatobiliary function and morphology in rats following alpha-naphthylisothiocyanate (ANIT) administration. Toxico1 Appl Pharmacol 119: 108-114, 1993.

21. Golbar HM, Izawa T, Yano R, Ichikawa C, Sawamoto O, Kuwamura M, Lamarre J and Yamate J: Immunohistochemical characterization of macrophages and myofibroblasts in $\alpha$-Naphthylisoth- iocyanate (ANIT)-induced bile duct injury and subsequent fibrogenesis in rats. Toxicol Pathol 39: 795-808, 2011.

22. Tarantino G, Finelli C, Colao A, Capone D, Tarantino M, Grimaldi E, Chianese D, Gioia S, Pasanisi F, Contaldo F, et al: Are hepatic steatosis and carotid intima media thickness associated in obese patients with normal or slightly elevated gamma-glutamyl-transferase? J Transl Med 10: 50, 2012. 
23. Tavian D, Degiorgio D, Roncaglia N, Vergani P, Cameroni I, Colombo R and Coviello DA: A new splicing site mutation of the ABCB4 gene in intrahepatic cholestasis of pregnancy with raised serum gamma-GT. Dig Liver Dis 41: 671-675, 2009.

24. Lurie Y, Webb M, Cytter-Kuint R, Shteingart S and Lederkremer GZ: Non-invasive diagnosis of liver fibrosis and cirrhosis. World J Gastroenterol 21: 11567-11583, 2015.

25. Matsui S, Yamane T, Takita T, Oishi Y and Kobayashi-Hattori K: The hypocholesterolemic activity of Momordica charantia fruit is mediated by the altered cholesterol- and bile acid-regulating gene expression in rat liver. Nutr Res 33: 580-585, 2013.

26. Soroka CJ, Mennone A, Hagey LR, Ballatori N and Boyer JL: Mouse organic solute transporter alpha deficiency enhances renal excretion of bile acids and attenuates cholestasis. Hepatology 51: 181-190, 2010.

27. Geenes V, Lövgren-Sandblom A, Benthin L, Lawrance D, Chambers J, Gurung V, Thornton J, Chappell L, Khan E, Dixon $\mathrm{P}$, et al: The reversed feto-maternal bile acid gradient in intrahepatic cholestasis of pregnancy is corrected by ursodeoxycholic acid. PLoS One 9: e83828, 2014

28. Poupon R: Ursodeoxycholic acid and bile-acid mimetics as therapeutic agents for cholestatic liver diseases: An overview of their mechanisms of action. Clin Res Hepatol Gastroenterol 36 (Suppl 1): S3-S12, 2012.

29. Ozel Coskun BD, Yucesoy M, Gursoy S, Baskol M, Yurci A, Yagbasan A, Doğan S and Baskol G: Effects of ursodeoxycholic acid therapy on carotid intima media thickness, apolipoprotein $\mathrm{A} 1$, apolipoprotein $\mathrm{B}$, and apolipoprotein B/A1 ratio in nonalcoholic steatohepatitis. Eur J Gastroenterol Hepatol 27: 142-149, 2015.

30. Kim MD, Kim SS, Cha HY, Jang SH, Chang DY, Kim W, Suh-Kim H and Lee JH: Therapeutic effect of hepatocyte growth factor-secreting mesenchymal stem cells in a rat model of liver fibrosis. Exp Mol Med 46: e110, 2014.

31. Friedman SL: Hepatic fibrosis-overview. Toxicology 254: 120-129, 2008

32. Suk KT, Kim DY, Sohn KM and Kim DJ: Biomarkers of liver fibrosis. Adv Clin Chem 62: 33-122, 2013.
33. El-Mezayen HA, Habib S, Marzok HF and Saad MH: Diagnostic performance of collagen IV and laminin for the prediction of fibrosis and cirrhosis in chronic hepatitis $\mathrm{C}$ patients: A multicenter study. Eur J Gastroenterol Hepatol 27: 378-385, 2015

34. Lee HH, Seo YS, Um SH, Won NH, Yoo H, Jung ES, Kwon YD, Park S, Keum B, Kim YS, et al: Usefulness of non-invasive markers for predicting significant fibrosis in patients with chronic liver disease. J Korean Med Sci 25: 67-74, 2010.

35. El-Shabrawi MH, Zein El Abedin MY, Omar N, Kamal NM, Elmakarem SA, Khattab S, El-Sayed HM, El-Hennawy A and Ali AS: Predictive accuracy of serum hyaluronic acid as a non-invasive marker of fibrosis in a cohort of multi-transfused Egyptian children with $\beta$-thalassaemia major. Arab J Gastroenterol 13: 45-48, 2012.

36. Nath NC, Rahman MA, Khan MR, Hasan MS, Bhuiyan TM, Hoque MN, Kabir MM, Raha AK and Jahan B: Serum hyaluronic acid as a predictor of fibrosis in chronic hepatitis $\mathrm{B}$ and $\mathrm{C}$ virus infection. Mymensingh Med J 20: 614-619, 2011

37. Nobili V, Alisi A, Torre G, De Vito R, Pietrobattista A, Morino G De Ville De Goyet J, Bedogni G and Pinzani M: Hyaluronic acid predicts hepatic fibrosis in children with nonalcoholic fatty liver disease. Transl Res 156: 229-234, 2010.

38. Li ZX, He Y, Wu J, Liang DM, Zhang BL, Yang H, Wang LL, Ma Y and Wei KL: Noninvasive evaluation of hepatic fibrosis in children with infant hepatitis syndrome. World J Gastroenterol 12: 7155-7160, 2006

39. Paumgartner G and Beuers U: Ursodeoxycholic acid in cholestatic liver disease: Mechanisms of action and therapeutic use revisited. Hepatology 36: 525-531, 2002.

40. Alaca N, Özbeyli D, Uslu S, Şahin HH, Yiğittürk G, Kurtel H, Öktem G and Çağlayan Yeğen B: Treatment with milk thistle extract (Silybum marianum), ursodeoxycholic acid, or their combination attenuates cholestatic liver injury in rats: Role of the hepatic stem cells. Turk J Gastroenterol 28: 476-484, 2017. 